\title{
Relationship between the tensile properties of articular cartilage from the human knee and age
}

\author{
G. E. KEMPSON \\ From the Department of.Medical Engineering, Level D, Centre Block, Southampton General Hospital, \\ Southampton SO9 $4 X Y$
}

SUMmARY The relationship between the tensile properties of articular cartilage and age has been investigated in vitro in the human knee joint. Specimens orientated parallel to the articular surface were excised from the superficial and deep zones of the femoral condyles of knee joints of persons in the age range from 8 to 91 years. The results showed that the tensile strength of the superficial zone increased with age to reach a maximum value in the third decade. Thereafter the strength decreased markedly with increasing age. The tensile strength of cartilage from the deep zone did not show an increase in the early years but decreased continuously with age. The tensile stiffness of the superficial layer at stresses of $5 \mathrm{MN} / \mathrm{m}^{2}$ and $10 \mathrm{MN} / \mathrm{m}^{2}$ increased to maximum values in the third decade and thereafter decreased with increasing age. The stiffness of the deep zone decreased continuously with age. It is suggested that these results reflect changes in the organisation of the collagen fibre mesh with age and possibly also changes in the collagen cross-links.

Osteoarthrosis is often referred to as a disease of aging, a statement which is supported by statistical evidence that the prevalence of the condition increases markedly with increasing age, reaching a maximum in the seventh decade. This raises the question whether changes occur in synovial joints with age which predispose to the development of osteoarthrosis.

One of the earliest features of the development of osteoarthrosis is degeneration of the articulating surfaces of the joint. This is characterised by fibrillation of the articular cartilage, in which the mesh of collagen fibres is disrupted. At this stage the cartilage matrix is fragmented and consequently mechanically weak. One of the pressing questions relating to the pathogenesis of osteoarthrosis is the sequence of events which leads to the initial fibrillation of cartilage.

Evidence exists which suggests that mechanical factors play a role in the development of osteoarthrosis. For example, McDevitt and Muir ${ }^{1}$ showed that, as a result of sectioning the anterior cruciate ligament of the canine knee, the dogs developed degenerative changes in the articular cartilage as soon as 3 weeks postoperatively, and these changes progressed to osteoarthrosis. Control tests revealed that the only

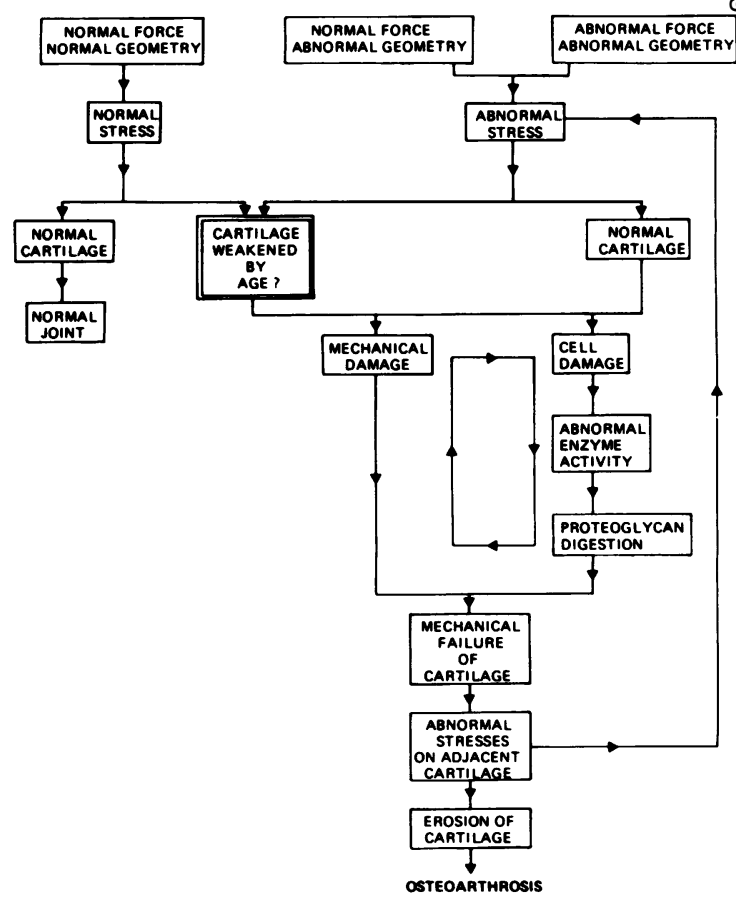

Fig. 1 Chart showing the possible role of mechanical factors in the development of osteoarthrosis. 
result of sectioning the cruciate ligament was to produce mechanical instability in the knee joint.

Fig. 1 shows a chart which indicates the possible routes by which mechanical factors may contribute to the development of osteoarthrosis. It is clear that if the mechanical properties of articular cartilage were to deteriorate with increasing age then mechanical damage would be more likely to occur.

The present investigation was performed to determine the relationship between the tensile properties of human articular cartilage and age.

\section{Materials and methods}

Articular cartilage was obtained from the femoral condyles of 24 knee joints in the age range from 8 to 91 years. All the joints were obtained from routine post-mortem examinations in which the cause of death was unlikely to affect the articular cartilage. They were then stored at $-20^{\circ} \mathrm{C}$ prior to testing.

The techniques for excising the cartilage from the joint surface and for preparing miniature tension specimens in the shape of a dumbell $200 \mu \mathrm{m}$ thick by $10 \mathrm{~mm}$ long with a central, parallel-sided, gauge length $0.5 \mathrm{~mm}$ wide by $3 \mathrm{~mm}$ long, have been described previously. ${ }^{23}$ Specimens were excised from 3 regions on each of the 2 femoral condyles, namely, from the patellar groove and from the central and posterior regions. The specimens, which were orientated with their axes parallel to the predominant alignment of the collagen fibres in the superficial zone, ${ }^{1}$ were prepared from the superficial and deep layers $(800 \mu \mathrm{m}$ below the surface). Only those specimens which had normal surfaces according to the Indian ink test of Meachim ${ }^{4}$ were tested.

Each specimen was immersed in Ringer's solution and tested in the plane parallel to the articular surface with a materials testing machine (Instron 1115). The test was performed at a constant rate of extension of 5 $\mathrm{mm} / \mathrm{min}$, and the tensile force applied to the specimens was recorded continuously by the load cell (Instron 2511-102).

The gauge length of $3 \mathrm{~mm}$ was marked on the specimen with 2 fine hairs attached transversely to the long axis, and measurements of the extension of the gauge length were made by a cinephotographic technique initially and later a closed circuit television system. In the first method a cine camera was used to photograph the gauge length continuously during the tension test. Tensile strains were determined from the extension of the gauge length divided by its initial length. The second method employed a closed circuit television extensometer (Hampton Video Systems Ltd SP123) in which the gauge marks were viewed by a closed circuit television camera connected to a target locator. This system determined the distance separating the gauge marks by a scanning technique. The output from the locator was fed into the $X$ axis of an $\mathrm{X}-\mathrm{Y}$ plotter (Hewlett-Packard 7044A), while the output from the tension load cell was fed into the $\mathrm{Y}$ axis. By suitable calibration the output was obtained in the form of a continuous curve of stress versus strain, and values of the tensile stiffness were determined from the gradient to the curve at 2 levels of stress, namely, $5 \mathrm{MN} / \mathrm{m}^{2}$ and $10 \mathrm{MN} / \mathrm{m}^{2}$. The results are shown graphically in Figs 2 to 5 .

\section{Results}

Fig. 2 shows the relationship between the tensile fracture stress and age for specimens from the superficial layer of the medial and lateral femoral condyles. The results show that the fracture stress increased initially to reach a maximum value around the middle of the third decade and thereafter decreased with increasing age. The linear regression coefficients for the regions of the graph from 0 to $24 \mathrm{yr}$ and from $24 \mathrm{yr}$ onwards were 0.5 and -0.86 respectively, indicating statistical significance.

Fig. 3 shows the relationship between fracture stress and age for specimens from the fourth layers of

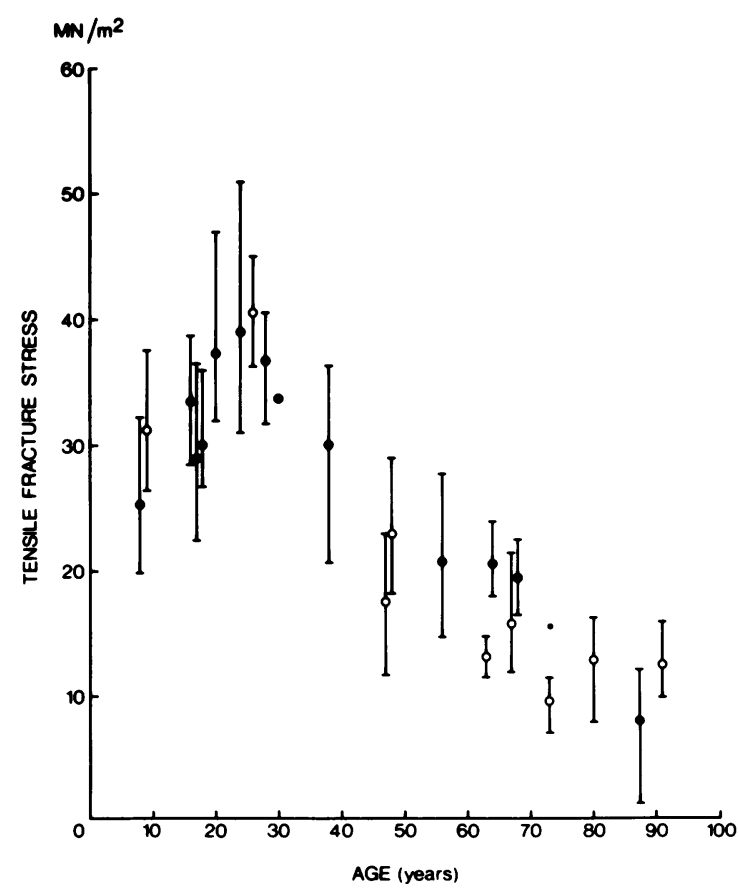

Fig. 2 Tensile fracture stress versus age for superficial zone specimens from the medial and lateral femoral condyles. Males -๑-. Females -०-. 
the medial and lateral condyles. At this level the fracture stress decreased continuously with increasing age without the initial increase seen in the superficial layer. The linear regression coefficient was $-0 \cdot 81$, which was statistically significant.

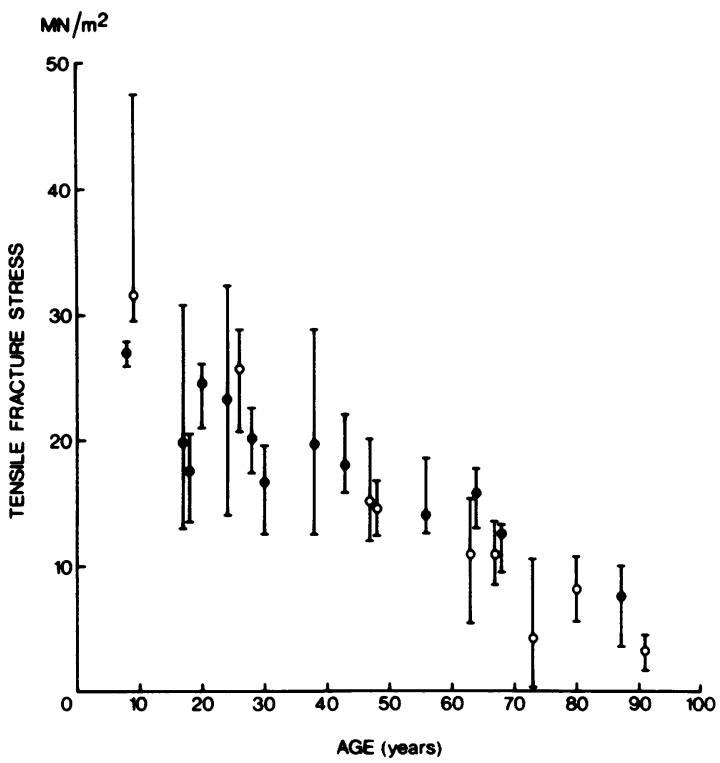

Fig. 3 Tensile fracture stress versus age for deep zone specimens ( $800 \mu \mathrm{m}$ below the surface) from both femoral condyles. Males -๑-. Females - - .
Figs 4 and 5 show tensile stiffness, at the stress of $10 \mathrm{MN} / \mathrm{m}^{2}$ and $5 \mathrm{MN} / \mathrm{m}^{2}$ respectively, versus age for specimens from the superficial layer of the medial and lateral condyles. At both stresses the stiffness increased to a maximum value by the middle of the

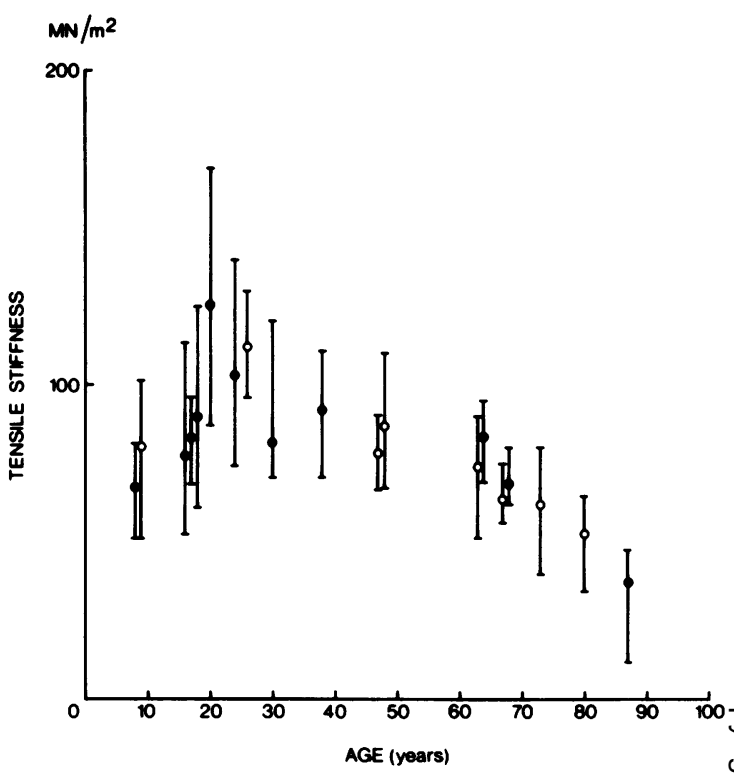

Fig. 5 Tensile stiffness at $5 \mathrm{MN} / \mathrm{m}^{2}$ versus age for superficial zone specimens from both femoral condyles. Males - $\bullet$-. Females - - -

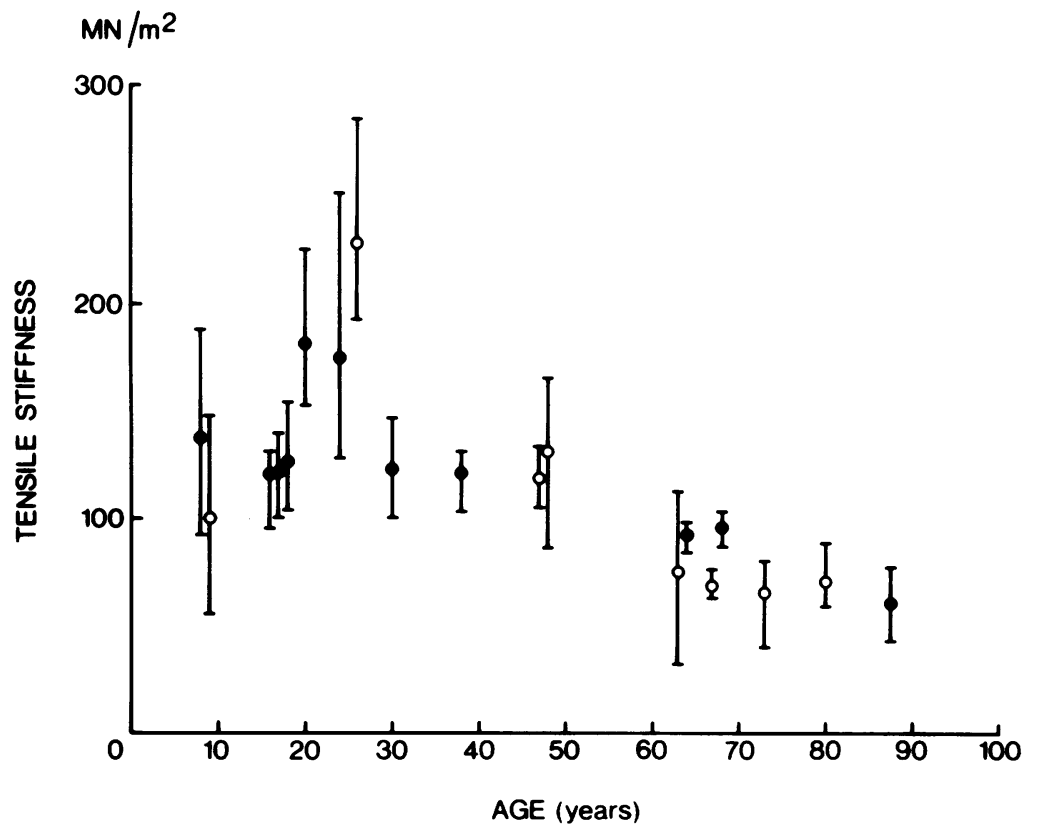

Fig. 4 Tensile stiffness at 10 $M N / \mathrm{m}^{2}$ versus age for super ficial zone specimens from both femoral condyles. Males $\rightarrow \bullet$. Females -O-. 
third decade and thereafter decreased with age. The linear regression coefficients for the 2 graphs in the region 0 to 24 yr were 0.61 and 0.47 respectively and for the region from 24 yronwards $-0 \cdot 81$ and $-0 \cdot 72$.

\section{Discussion}

It is clear from the present study that the tensile properties of human articular cartilage from the femoral condyles of the knee deteriorated with increasing age from the middle of the third decade. As further evidence of age related changes in the tensile properties of cartilage Weightman ${ }^{5}$ showed that the fatigue resistance of femoral head cartilage in tension decreased with age. The rate of decrease was greater than that predicted from younger specimens, from which it was concluded that another mechanism distinct from fatigue was responsible. It is clear therefore that the deterioration in the tensile properties of cartilage with increasing age from the middle of the third decade reflects either a decrease in the amount of collagen in the matrix or changes in the organisation and integrity of the collagen mesh.

The first suggestion can be discounted, since it has been shown that the collagen content of cartilage on the femoral head does not decrease with age.$^{6}$ On the second point preliminary results in the author's laboratory ${ }^{7}$ have revealed that the organisation of the collagen fibrils became more widely spaced and larger in diameter. It is tempting to suggest, therefore, that organisational changes in the collagen mesh contribute to the observed decrease in tensile strength and stiffness.

Recent results from the author's laboratory ${ }^{8}$ showed that human leucocyte elastase considerably reduced the tensile strength and stiffness of articular cartilage. The associated release of collagen was less than $1 \%$ and the specimens appeared normal under the light microscope.

It is known that leucocyte elastase cleaves the nonhelical terminal peptides of tropocollagen molecules, thereby disrupting the major intra- and intermolecular cross-links. ${ }^{9}$ It is tempting to suggest, therefore, that modification of the collagen crosslinks occurs in articular cartilage with increasing age, which contributes to the deterioration in the tensile properties.

Robins et al. ${ }^{10}$ have shown that the major reducible cross-links in bovine skin decrease with age until they disappear at maturity. The behaviour was stated to be similar in cartilage, so that in man the reducible cross-links disappear between the ages of 17 and $20 \mathrm{yr}$. There is no evidence that the reducible crosslinks are converted to nonreducible cross-links as a function of age. However, it may be that changes in the cross-links with age are reflected in the tensile properties of cartilage.

\section{References}

${ }^{1}$ McDevitt C A, Muir $\mathrm{H}$. Biochemical changes in the cartilage of the knee in experimental and natural osteoarthrosis in the dog. $J$ Bone Joint Surg 1976; 58B: 94-101.

${ }^{2}$ Kempson G E, Muir H, Pollard C, Tuke M. The tensile properties of the cartilage of human femoral condyles related to the content of collagen and glycosaminoglycans. Biochim Biophys Acta 1973; 297: 456-72.

${ }^{3}$ Kempson G E. Mechanical properties of articular cartilage and their relationship to matrix degradation and age. Ann Rheum Dis 1975; 34: suppl 2: 111-4.

4 Meachim G. Light microscopy of Indian ink preparations of fibrillated cartilage. Ann Rheum Dis 1972; 31: 457-64.

5 Weightman B O. Tensile fatigue of human articular cartilage. J Biomech 1976; 9: 193-200.

- Venn M F. Variation of chemical composition with age in human femoral head cartilage. Ann Rheum Dis 1978; 37: 168-74.

7 Knight D P, Kempson G E. The relationship between the tensile properties of human articular cartilage and age.J Bone Joint Surg 1981; 63B: 641-2.

${ }^{8}$ Bader D L, Kempson G E, Barrett A J, Webb W. The effects of leucocyte elastase on the mechanical properties of adult human articular cartilage in tension. Biochim Biophys Acta 1981; 677: 103-8.

- Barrett A J. The possible role of neutrophil proteinases in damage to articular cartilage. Agents Actions 1978; 8: 11-8.

10 Robins S P, Shimokomaki M, Bailey A J. The chemistry of the collagen cross-links: age related changes in the reducible components of intact bovine collagen fibres. Biochem J 1973; 131: 771-80. 